\title{
Carcinoma of Stomach diagnosed by Trans abdominal Sonography and guided FNAC where Endoscopy was not conclusive-Three case reports.
}

\author{
K Z Shah ${ }^{1}$, N Begum ${ }^{2}$, S M Badruddoza ${ }^{3}$, P Ahmed $^{2}$, \& M Hossaon ${ }^{2}$
}

\begin{abstract}
Tranabdominal sonography along with USG guided FNAC can effectively evaluate the case of gastric carcinoma; while conventional endoscopy of upper gastrointestinal tract or Barium meal X-ray may not diagnose that case coclusively. Moreover, tranabdominal sonography is comparatively more cost effective and has also no radiation effect. From this perspective, this study highlights three case reports where endoscopy of upper gastrointestinal tract could not conclude the diagnosis but tranabdominal sonography along with USG guided FNAC could delineate the condition conclusively-which were later confirmed by histopathological examination.
\end{abstract}

TAJ 2013; 26: 98-102

\section{Introduction}

The incidence of the gastric carcinoma varies considerably in different parts of the world. It is frequent in Japan, China and Southern Europe but relatively uncommon in USA. It has been suggested that nitrites, often used as preservatives in food and present in drinking water, can be converted into the carcinogens nitrosamines in the milieu of the stomach.

The geographical incidence of $\mathrm{H}$. Pylori, leading to type $\mathrm{B}$ gastritis parallels that to gastric cancer.

Assessment of stomach is not commonly includes routine scanning protocol of upper abdominal Ultrasound (USG). However assessment of stomach in patients presenting with epigastric pain can yield valuable result.

Ultrasound is often used as the first imaging modality in a large variety of abdominal complaints and clinically unsuspected gastric carcinomas and may image this condition at first. This paper presents three cases of carcinoma of stomach detected by trans-abdominal Ultrasound along with USG guided FNAC; where endoscopy findings and histopathology was negative. Finally partial gastectomy followed by histopathology confirmed the diagnosis.

\section{Case-1:}

A 59 yrs old lady came to our Institute for USG of upper abdomen with complaint of severe upper abdominal pain, dyspepsia and weight loss. Before sonography she went through several routine investigation and endoscopy. Her report revealed neutrophlic leukocystosis \& high ESR. Endoscopy and histopathology report was not conclusive.

Ultrasound revealed a complex tubular structure in upper abdomen especially in epigastric region. Lumen of the pylorus was not well delineated, however bright linear structure was present

${ }^{1}$ Director, Institute of Nuclear Medicine \& Allied Sciences, Rajshahi, Bangladesh.

${ }_{2}$ Assistant Professor (SMO), Institute of Nuclear Medicine \& Allied Sciences, Rajshahi, Bangladesh

${ }^{3}$ Professsor \& Head of the Dept of Pathology, Rajshahi Medical College, Bangladesh 
centrally with solid hypoechoic periphery. Visualized part of stomach was filled with fluid and food particles. (Fig-II) Then patient was gone through USG guided FNAC (Fine Needle Aspiration Cytology) guidence of ultrasound transducer. Needle was probed to stated mass and tissue was taken for cytopathology by an expert Histopathologist. FNAC revealed carcinoma of the stomach involving pylorus. Finally patient was operated; partial gastectomy done. Tissue was sent for biopsy which revealed Adenocarcinoma of the stomach.

\section{Case-2:}

A 60 yrs female came for abdominal Ultrasonography by a Gastro-enterologist

to evaluate upper abdominal lump. USG was done in empty stomach using $3.5 \mathrm{MHz}$ Curvilinear sectoral probe of EUS-405 Hitachi Ultrasound machine.

USG report revealed an elongated complex epigastric lesion with centrally linear echogenic area and solid hypoechoic periphery. It was placed anterior to pancreas and superimposed over left hepatic parenchyma however separated from them. Mild ascites was seen in peritoneal cavity. Few small echogenic focal lesions were detected in right hepatic lobe; USG feature were suggestive of pyloric neoplasm with hepatic metastases.

Other investigation showed microcytic hypochromic anaemia, Hb-5.80 gm/dl, and ESR$10 \mathrm{~mm}$ in $1^{\text {st }}$ hour (westergren), mild neutrophilic leuckocystosis with normal RBS and S. creatinine levels.

The Endospic examination was done and the report was not conclusive. It revealed Gastritis (Malignancy could not excluded), Fig- III-shows endoscopic picture.

Then USG guided FNAC had been advised, one Institute revealed no malignancy but another suggested pyloric growth

Then patient was operated, during laparatomy there was huge diffuse

malignancy involving stomach mainly pyloric canal, antrum \& lower part of body with peritoneal involvement. The mass was removed; near total gastectomy done.

Tissue was sending for histopathology and the report revealed diffuse undifferentiated carcinoma of the stomach. After surgery chemotherapy was given.

\section{Case-3:}

A 45 yrs old man was suffering from epigastric pain, sudden weight loss, vomiting

\& dyspepsia. No H/O malena or haematemasis. On examination tenderness

was present over epigastric region otherwise no palpable mass felt. Initially patient went through blood examination \& upper GIT endoscopy. Blood reports were high ESR and neutrophilic leukocytosis. Endoscopy procedure was not conclusive due to patient's non- cooperation.

The patient was send to our Institute for Nuclear Medicine \& Ultrasound Rajshahi for abdominal sonography.

Sonography showed thin tubular structure measuring about $5.8 \times 2.8 \mathrm{~cm}$ seen in

epigastrium. The structure seems to be thick pylorus and lumen almost obliterated. To exclude luminal stenosis fluid filled stomach was evaluated during drinking water and after 30 minutes that revealed water was not passing to the pyloric

region during procedure and after. USG report was given as thick tubular lower end of stomach (suspected pyloric neoplasm).

Again he was seen for sonography guided FNAC, by using $3.5 \mathrm{MHz}$ curvillinier transducer of Sonoline G60S Ultrasound systems of SIEMENS mechine, tissue was taken from its wall.

Cytologically diagnosis was not conclusive, however significant atypical cells present and advised for open biopsy.

Laparotomy was performed. Elongated hard mass was seen in lower end of stomach,

partial gastectomy done. No enlarged celiac group of lymph nodes was detected. No peritoneal involvement or organ metastases were present. . 
Finally histopathology report reveals Adenocarcinoma of the stomach involving pylorus.

\section{Discussion:}

The incidence of the gastric carcinoma varies considerably in different parts of the world. It is frequent in Japan, China and Southern Europe but relatively uncommon in USA. It has been suggested that nitrites, often used as preservatives in food and present in drinking water, can be converted to the carcinogens nitrosamines in the milieu of the stomach.

The geographical incidence of $\mathrm{H}$. Pylori, leading to type B gastritis parallels that to gastric cancer. ${ }^{1}$

Assessment of stomach is not commonly includes routine scanning protocol of upper abdominal Ultrasound (USG). However assessment of stomach in patients presenting with epigastric pain can yield valuable result. ${ }^{2}$

Ultrasound is often used as the first imaging modality in a large variety of abdominal complaints and clinically unsuspected gastric carcinomas may image first by it. ${ }^{3}$

The Normal apperance of the stomach illustrated three distinctive layers that can usually be seen in a transabdonimal ultrasound, The mucosca \& submucosa appears hyperechoic, muscularis Propria is hypoechoic \& subsererasa is hyperechoic. ${ }^{4}$ Fig- (I) shows normal apperance of pylorus.

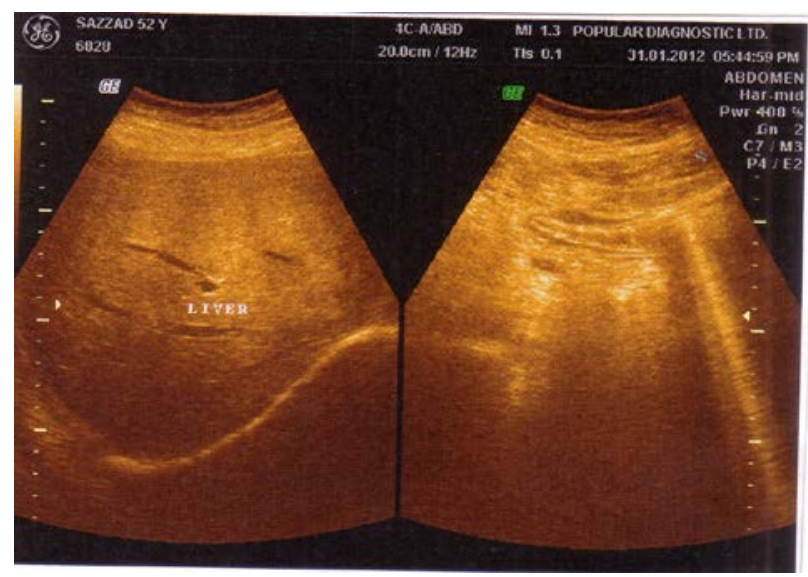

Fig - I - Normal pylorus
Loss of wall stratification has shown to be a sign of gastric malignancy. This may be accounted for by the invasiveness of the disease process itself, in which the tumour invades across different layers. Gastric wall thickening without loss of wall stratification favours a benign process, such as gastric ulcer, Menetrier's disease and anisakiasis. Apart from loss of wall stratification, the degree of gastric wall thickening also gives a clue to the nature of underlying disorder. The songraphic thickness of normal gastric body and antral wall measures upto $5 \mathrm{~mm}$ in a non-distended state. ${ }^{5}$

In a healthy population, visualization of the gastric autrum has been reported to be upto $100 \%$, while the body and fundus is less consistently seen. ${ }^{6}$

Transabdominal ultrasound examination has also been shown to have efficacy in visualising gastric carcinoma $^{7}$

In this paper all three cases were initially diagnosed by trans abdominal sonography followed by USG guided FNAC and histopathology.

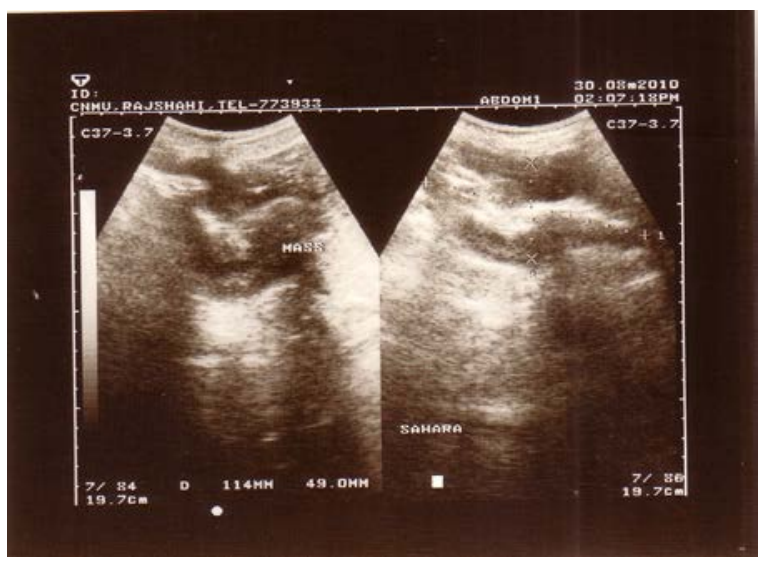

Fig- II

Gastric carcinoma, in contrast, arises from the gastic mucosa is usually more echogeuic, tends to invade vertically and evaluation of gut wall pathology on Sonography is important particularly if associated with abnormality of the perienteric soft tissues, creates a mass effeet, easily seen on ultrasonograph. Characterstic sonographic pattern gut wall pathology is familiar target pattern. ${ }^{8}$ 
Bluth et al., referred to the pattern as a "pseudokidney".In this pattern, there is hypoechoic peripheral rim corresponds to thickened gut wall, where as the echogenic center relates to residual gut lumen ${ }^{9}$

In our cases two target type lesions were seen but in another it appeared ill-defined and irregular.

In a study it is stated that Gastric Submucosal tumors (SMTs) can also be visulized using transabdonimal ultrasound of water-filled stomach. This non-invasive method may be potentially useful for surveillance in patient with SMTs ${ }^{10}$

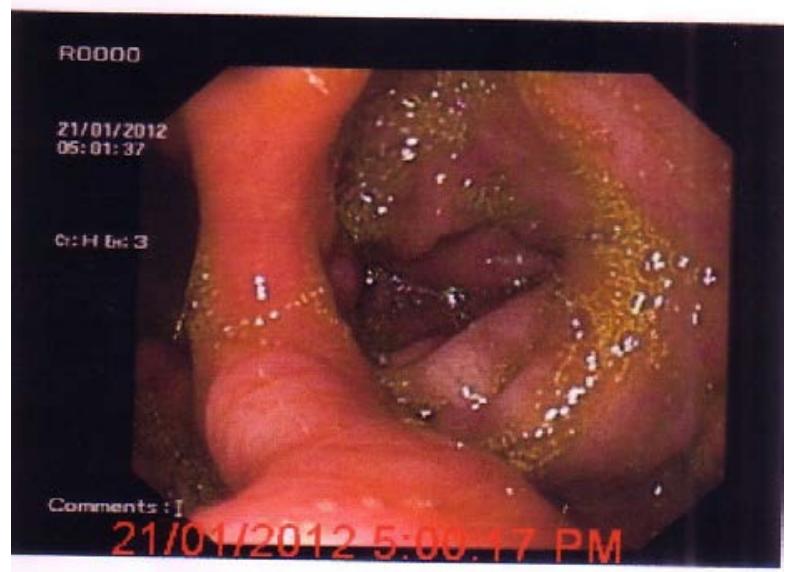

Fig- III

In Sonography carcinoma of stomach show complete distruption of wall layering and presence of heterogenous intraluminal masses. ${ }^{11}$

In this report of three cases, masses were present.

Which are in concordance with that of Yeh \& Rabiaowitz.(1981) Who stated that sonographic features of gastric tumours can be divided into three main categories.

a) Thickend gastric wall due to infitration by tumour

b) A mass

c) A combination of two. ${ }^{12}$

In another study by S. Singh, et al., in Scirrhous carcinoma of the stomach,diffuse circumferential wall thickening was seen in sonograpy. Endoscopy interpretated case as normal. Owing to the diffuse wall thickening seen on sonography, lymphoma was considered in the differential diagnosis in addition to diffuse carcinoma and repeat endoscopy was undertaken. Repeat endoscopic biopsy and histopathology was negative. ${ }^{13}$

In our all cases endoscopic findings were negative for malignancy but sonography report \& USG guided FNAC revealed carcinoma stomach.

Sonography underestimated the exogastric extent of gastric carcinoma but majority of the cases not available by endoscopy. Well known difficulty in endoscopy in the diagnosis these tumours are due to overlying normal mucosa. The diagonostic yield is higher in exophytic lesions than in infiltrative lesions. ${ }^{14}$

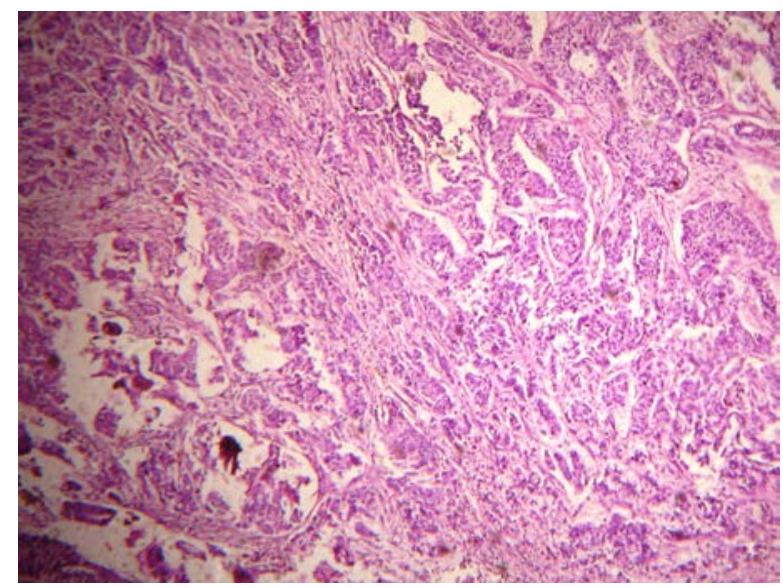

Fig: 1V- Histopathological Section Showing Infiltrating Adenocarcinoma H\&E $\times 400$

\section{Conclusion :}

Evaluation of gastric carcinoma by tranabdominal sonography \& USG guided FNAC allow unique \& detailed evaluation of gastric wall layers not availble either by endoscopy or Ba-meal. Loss of wall layering suggest malignant lesion. Intraluminal abnormalities and adjacent mass are well demonestrated by sonography, allow better evaluation of extent of lesion, where endoscopy can't negotiate proximal growth or area of narrowing. The USG along with USG guideded FNAC are procedure to evalute gastric carcinoma in a cost effective manner without any radiation hazard. 


\section{Reference:}

1. D.J. C. Shearman, Diseases of the alimentary tract and pancreas, DAVIDSON'S

principles and practice of Medicine, C.R.W. Edwards, I.A.D Bouchier, C.Haslett,

E.R.Chilvers $17^{\text {th }}$ ed. Churchill Livingstone, 1995:7,435.

2. MFE Wong, Wk chau \& CS Eheng. Carcinoma of stomach detected by routine trans abdominal Ultrasound, Biomedical Imagine and Intervention journal, 2010, Oct-Dec, 6(4): e39.

3. S. Singh, V.Chowdhury, Efficacy of high resolution Transabdominal Sonography

of fluid filled stomach in the evaluation of gastric carcinoma, J radiol Imag,

2005; 15:4 421-426.

4. Okonobu H, Hata J, Haruna K, Hara M, Nakamura K, Tanaka S, Chayama K, Giant Gastric Folds : Differential Diagnosis at Us1. Radiology, 2003; 226(3) : 686-690.

5. Fleischer Ac, Muhletaler CA, James AE, Jr Sonographic assessment of bowel wall.

Am J Roentgenol. 1981; 136 (5) : 887-891 [pubmed]

6. Perlas A, Chan WWS, LupuCM, Mitsakais N, Hanbidge A, Ultrasound Assessment of Gastric Coatent Volume. Anestuesiology 2009; 111 (1) : 82-89.
7. Singh S, Chowdhury V. Efficacy of high lesolution transabdominal sonography of the fluid filled stomach in the evaluation of gastric carcinomas, Indian Journal of Radiological imaging. 2005; 15(4) | 421-426.

8. Lutz H, Petzoldt R : Ultrasonic patterns of space occupying leusions of the stomach \& the intestine. Ultrasound Med Biol 1976, 2:129-131.

9. Bluth El, Merritt CRB, Sullivan MA. Ultrasonic evaluation of the stomich, small bowel \& colon. Radiology 1979, 133:677-680.

10. M. Polkowski, J. Palucki, E. butruk. Transalyc, Ultrasound for visualizing Gastric Submucosal Tumors Dignosed by Endosonography : can surveillance Be simplified. Thieme eJournals Endoscopy 2002; 34(12): 979-983.

11. Stephanic, R. Wilson, The gastrointestinal fract, Diagonostic Carol M. Rumack, MD, S.R. Wilson, MD. J. William. Charboneau, Jo-Ann-M. Johnson Diagnostic Ultrasound, $3^{\text {rd }}$ edition Mosby Inc 2005; 8: 269-312.

12. Yeh HC, Robinowitz JC, Ultrasonographic \& Competened Tomography of gastric wall lea--Radiology 1981; 141: 147-155.

13. S. Singh, V chowdhury, Efficiancy of high resolution Transabdominal sonogramy et at., of full filled stomach if te evaluation gos can class IJRI, 2005: Nov : Tue 15:4(67) 421- 426

14. Winawer SJ, Posner G, Lightdale CJ, Sherlock P, Melaned M, Fortner JG, Endoscopic diagonestic in advanced gastric cancer. Factors infencing yield, Gastro-enterology 1975; 69 : 1183-1187.

Dr Md Kabiruzzaman Shah M Phil (NM), IAEA Fellow UCL (London UK) Director Institute of Nuclear Medicine \& Allied Sciences, Rajshahi, Bangladesh Email-shahkabirz@gmail.com 